\title{
Análisis comparativo de los valores de las presiones respiratorias máximas con los valores de referencia en una población adulta sana
}

\author{
Comparative analysis of maximal respiratory pressures with the reference values \\ of a healthy adult population
}

\author{
A. Lista-Paz ${ }^{\mathrm{a}, \mathrm{b}_{*},}$ S. Souto Camba ${ }^{\mathrm{a}, \mathrm{b}}$, J. Vilaró Casamitjana $^{\mathrm{c}}$, A. Quintela-del-Río ${ }^{\mathrm{a}}$ \\ A. López García ${ }^{\mathrm{a}, \mathrm{b}}$ y L. González Doniz \\ ${ }^{a}$ Facultad de Fisioterapia, Universidade da Coruña, España \\ ${ }^{b}$ Grupo de investigación en intervención psicosocial y rehabilitación funcional, Universidade da Coruña, \\ España \\ ${ }^{c}$ Facultad de Ciencias de la Salud Blanquerna, Global Research on Wellbeing (GRoW), Universitat Ramón \\ Llull, Barcelona, Espana
}

\begin{abstract}
Resumen
Antecedentes y objetivo. La determinación de las presiones respiratorias máximas es un procedimiento no invasivo de gran utilidad clínica para la evaluación de la fuerza de los músculos respiratorios. El objetivo es analizar en qué medida las ecuaciones predictivas existentes para población española, se ajustan a los valores observados de presión inspiratoria y espiratoria máximas (PEM y PIM) en una muestra de sujetos adultos sanos.

Material y métodos. Estudio descriptivo observacional de corte transversal en el que se reclutaron 63 sujetos sanos mediante muestreo probabilístico aleatorizado simple entre la comunidad universitaria de la Universidade da Coruña. Las presiones respiratorias máximas se efectuaron con un transductor de presiones conectado a una boquilla de submarinista, siguiendo las recomendaciones de la Sociedad Española de Neumología y Cirugía Torácica (SEPAR). Se compararon los resultados con las ecuaciones de Morales de 1997.

Resultados. Se presentan los datos de 24 mujeres y 39 hombres (45,94 $\pm 16,71$ años). Existe una diferencia estadísticamente significativa en las mujeres de $-19,08 \pm 23,57 \mathrm{cmH}_{2} \mathrm{O}$ y de $-28,13 \pm 29,93 \mathrm{cmH}_{2} \mathrm{O}$ para la PIM y la PEM, respectivamente, entre el valor observado y el valor predicho. Asimismo, en el caso de los hombres se observó una diferencia estadísticamente significativa de $-25,18 \pm 24,31 \mathrm{cmH}_{2} \mathrm{O}$ para la PIM y de $39,53 \pm 44,38 \mathrm{cmH}_{2} \mathrm{O}$ para la PEM.

Conclusiones. Las ecuaciones predictivas disponibles para las presiones respiratorias máximas sobreestiman considerablemente los valores alcanzados de la PIM y la PEM. Los resultados del presente estudio ponen de manifiesto la necesidad de realizar nuevas ecuaciones de referencia a través de un estudio multicéntrico representativo de toda la población española.
\end{abstract}




\begin{abstract}
Background and aim. The determination of maximal respiratory pressures is a non-invasive process of high clinical value for assessing respiratory muscle strength. The aim is to analyse to what extent the existing predictive equations for the Spanish population are adjusted to the maximal inspiratory and expiratory pressures (MIP and MEP) values observed in a sample of healthy adult subjects.

Material and methods. A cross-sectional study was conducted on a sample of 63 healthy subjects recruited from the A Coruña University community using a simple random probabilistic method. Maximal respiratory pressures were performed using a pressure transducer connected to a scuba mouthpiece according to the standards of Spanish Society of Pulmonology and Thoracic Surgery (SEPAR). The results were compared with predictive equations proposed by Morales in 1997.

Results. The study included the results from 24 females and 39 males (45.94 \pm 16.71 years). Females showed a statically significant difference between the observed and predictive values, with $-19.08 \pm 23.57 \mathrm{cmH}_{2} \mathrm{O}$ and $-28.13 \pm 29.93 \mathrm{cmH}_{2} \mathrm{O}$ for MIP and MEP. A statistically significant difference was also observed for males with $-25.18 \pm 24.31 \mathrm{cmH}_{2} \mathrm{O}$ for MIP and $-39.53 \pm 44.38 \mathrm{cmH}_{2} \mathrm{O}$ for MEP.

Conclusions. Predictive equations to calculate the theoretical values of maximal respiratory pressures in a healthy Spanish adult population overestimate considerably the real values of MIP and MEP. The results of this study highlight the need to create new reference equations by conducting a multicentre study representative of the entire Spanish population.
\end{abstract}

\title{
Palabras clave
}

Presiones respiratorias máximas; Test de función respiratoria; Músculos respiratorios; Valores de referencia

Keywords

Maximal respiratory pressures; Respiratory function tests; Respiratory muscles; Reference values

\section{Introducción}

Las enfermedades que afectan al aparato respiratorio son cada vez más prevalentes según la Organización Mundial de la Salud ${ }^{1}$. La rehabilitación pulmonar es la intervención no farmacológica con mayor evidencia científica ${ }^{2}$. En la evaluación respiratoria previa tiene especial relevancia la valoración de la musculatura respiratoria. Existen diferentes situaciones que pueden cursar con disfunción de la musculatura respiratoria, entendida esta como la incapacidad de un músculo para cumplir su cometido ${ }^{3}$, pudiendo deberse a un déficit de la fuerza, de la resistencia, o de ambos factores ${ }^{4}$. Las patologías que afectan a la musculatura respiratoria son principalmente la enfermedad pulmonar obstructiva crónica, enfermedades neuromusculares y degenerativas, el accidente cerebrovascular y aquellas que cursan con deformidades o alteraciones estructurales de la caja torácica ${ }^{5,6,7}$.

Los métodos de evaluación de la fuerza de la musculatura respiratoria más habituales en la práctica clínica son las presiones respiratorias máximas (PRM) obtenidas de forma voluntaria y no invasiva a través de la presión generada en boca, registrándose con un manómetro o transductor de presiones $^{4,8,9}$.

Para la interpretación de los resultados de las presiones inspiratorias e espiratorias máximas (PIM y PEM), además de los valores absolutos obtenidos en las PRM, diferentes investigaciones recientes han observado la necesidad de establecer una horquilla basada en valores relativos a los valores teóricos, puesto que la edad y las medidas antropométricas tienen una gran influencia en los resultados de la PIM y la PEM $^{10}$. Por ello, disponer de valores de normalidad en la población a estudiar es fundamental para poder interpretar correctamente los resultados de las pruebas, permitiendo la adecuada prescripción del entrenamiento de la musculatura respiratoria. 
Desde el año $1969^{11}$ diversos autores han publicado ecuaciones predictivas para el cálculo de los valores de referencia de las PRM. En España solo se dispone de las propuestas en 1997 por Morales et al. ${ }^{5}$, obtenidas en base a un estudio realizado en Valencia con 264 participantes. Las sociedades científicas internacionales, American Thoracic Society (ATS) y European Respiratory Society (ERS), recomiendan que los valores de referencia relacionados con la función pulmonar sean actualizados cada 10 años, ya que las características de la población de sujetos sin patología evolucionan debido a los cambios en la alimentación, el estado de salud, las condiciones medioambientales y otros factores que condicionan el llamado «efecto de cohorte» ${ }^{12}$. Por estos motivos creemos necesario revisar el grado de concordancia existente entre los valores observados en población adulta sana y los obtenidos mediante las citadas ecuaciones.

El presente estudio tiene como objetivo analizar en qué medida las ecuaciones predictivas existentes para población española para las PRM se ajustan a los valores observados en una muestra de sujetos españoles adultos sanos.

\section{Material y métodos}

Estudio descriptivo observacional de corte transversal, realizado en la Facultad de Fisioterapia de la Universidade da Coruña. Se reclutaron 63 sujetos sanos mediante muestreo probabilístico aleatorizado simple entre el personal y alumnado de la UDC. Se envió una carta de participación a todos los miembros de la comunidad universitaria de la UDC a través de la lista de distribución de correo electrónico, a la cual respondieron 324 sujetos. Entre las personas que se presentaron voluntarias, se realizó una selección aleatoria, a través de una tabla de números aleatorios generada por ordenador, de 63 sujetos.

Se incluyeron personas caucásicas, subjetivamente sanas, no fumadoras, con edad comprendida entre 18 y 83 años. Se excluyeron aquellos sujetos con historia de enfermedad respiratoria, neuromuscular, cerebrovascular y/o cardíaca; deformidades de la caja torácica; enfermedad respiratoria los 30 días previos; uso habitual de medicamentos antialérgicos, corticoides orales, depresores del sistema nervioso central, barbitúricos o relajantes musculares ${ }^{5,13}$; hernias abdominales; problemas agudos de oído medio; fístulas de la pared costal o pulmonares; glaucoma, desprendimiento de retina y/u operación de cataratas reciente; obesidad superior al $20 \%$ de sobrepeso; embarazadas y deportistas de élite ${ }^{5,9,14}$. Durante las pruebas, además, fueron excluidos los sujetos que mostraron una espirometría alterada según la interpretación propuesta por la SEPAR ${ }^{15}$ y aquellos incapaces de comprender las instrucciones para las pruebas.

Este estudio fue aprobado por el Comité de Ética de la Universidade da Coruña (número de expediente 2018-0014, aprobado el 5 de julio de 2018). Todos los participantes firmaron un consentimiento informado.

\section{Procedimiento para las mediciones}

Todas las mediciones fueron realizadas por la misma fisioterapeuta entrenada en la realización de estas pruebas. Se recabaron mediante entrevista personal estructurada los datos sociodemográficos, los antecedentes patológicos, historial tabáquico y tratamiento farmacológico habitual. Se registraron las medidas antropométricas (peso, talla e índice de masa corporal) y se objetivó la actividad física semanal a través de la versión corta del cuestionario internacional de actividad física, validado en idioma español (International Physical Activity Questionnaire- IPAQ short ${ }^{16}$. Seguidamente se realizó la evaluación de la función pulmonar, mediante espirometría forzada, según los estándares internacionales de la ATS/ERS ${ }^{17,18}$ así como los nacionales de la $\mathrm{SEPAR}^{15}$. Por último se llevó a cabo la medición de las PRM. 


\section{Presiones respiratorias máximas}

Para su determinación se siguieron las recomendaciones de la SEPAR, que están en consonancia con las empleadas por Morales et al. ${ }^{5,9}$. Las maniobras fueron efectuadas con el paciente en sedestación. Se utilizó un transductor digital de presiones con un rango de lectura de $\pm 300 \mathrm{cmH}_{2} \mathrm{O}$, una resolución de $1 \mathrm{cmH}_{2} \mathrm{O}$ y una exactitud del 3\% (modelo 511-8D0-MU1, Sibel Group, Barcelona, España) conectado a un espirómetro Datospir ${ }^{\circledR} 120 \mathrm{C}$. El tubo del transductor presentó un orificio de salida en su parte más distal, con un diámetro inferior a $1 \mathrm{~mm}$. Se empleó una boquilla de tipo submarinista, de goma rígida, con una arandela ancha, impidiendo las fugas de aire. Además, se colocaron pinzas nasales durante las mediciones.

Se comenzó por la determinación de la PEM, explicándole al sujeto en qué consiste la prueba, e indicándole cómo debe colocar sus manos sobre las mejillas para impedir las fugas de aire y las oscilaciones debidas a los músculos bucinadores, minimizando la contribución de los mismos. Tras una inspiración máxima, próxima a la capacidad pulmonar total, se cerró la llave bidireccional del transductor de presiones, y se solicitó al sujeto que soplase con todas sus fuerzas durante 3-5 segundos. Se motivó vigorosamente al participante para que realizase un esfuerzo máximo. Tras la medición de la PEM el sujeto descansó 5 min y, entre repetición y repetición de cada una de las presiones máximas reposó al menos un minuto.

Para la obtención de la PIM, tras una espiración máxima hasta el volumen residual se cerró la llave bidireccional del transductor, y se incentivó al sujeto para que inspirase con máximo esfuerzo durante 3-5 segundos.

Se realizaron los intentos necesarios (hasta un máximo de 10) para obtener 6 maniobras técnicamente correctas, en aras de evitar el efecto aprendizaje, el cual ha demostrado tener influencia sobre el resultado de la PIM y la PEM $^{10,19}$. Entre las 6 maniobras técnicamente correctas, se buscaron 3 reproducibles, con una variabilidad $<5 \%$. Se registró el mayor valor de las 3 maniobras aceptables y reproducibles.

\section{Análisis estadístico}

Tras comprobar la normalidad en la distribución de los datos mediante el estadístico ShapiroWilks, se empleó una prueba $t$ de Student para muestras apareadas con el objetivo de contrastar la existencia de diferencias significativas entre los valores observados y los calculados mediante las ecuaciones de referencia en la PIM y la PEM. Se aceptó un nivel de significación estadística de $\mathrm{p}<0,05$. Para el estudio de las correlaciones entre las PRM y las diferentes variables antropométricas, espirométricas y de actividad física, se empleó el coeficiente de correlación de Pearson. La categorización de Dancey y Reidy ${ }^{20}$ fue empleada para interpretar la fuerza de las correlaciones. Asimismo, se empleó una regresión cuadrática para explorar el comportamiento de la diferencia entre el valor observado y el valor predicho de las PRM en relación con la edad. El procesamiento y análisis de los datos se realizó con el paquete estadístico SPSS, versión 24.0 para Windows (Armonk, NY: IBM Corp.). 


\section{Resultados}

Se presentan los resultados de 63 sujetos distribuidos por sexos, dado que las ecuaciones predictivas se elaboran de este modo. La muestra está constituida por 24 mujeres $(43,3 \pm 17,8$ años) y 39 hombres (47,6 \pm 16 años). Las características de la muestra se reflejan en la tabla 1 . De los 63 sujetos que participaron en el estudio, un 3,17\% realizaba una actividad física semanal baja, un $44,44 \%$ moderada y un $52,38 \%$ alta (IPAQ-Short). Se observó una diferencia estadísticamente significativa de $-19,08 \pm 23,57 \mathrm{cmH}_{2} \mathrm{O}$ para las mujeres y de $-25,18 \pm 24,31 \mathrm{cmH}_{2} \mathrm{O}$ para los hombres entre la PIM hallada en nuestra muestra y la calculada mediante la ecuación predictiva (valor de referencia para la $\mathrm{PIM}=98,50 \pm 12,74 \mathrm{cmH}_{2} \mathrm{O}$ en mujeres y $131,10 \pm 17,78 \mathrm{cmH}_{2} \mathrm{O}$ en hombres). Asimismo, se observó una diferencia estadísticamente significativa de $-28,13 \pm 29,93$ $\mathrm{cmH}_{2} \mathrm{O}$ para las mujeres y de $-39,53 \pm 44,38 \mathrm{cmH}_{2} \mathrm{O}$ para los hombres entre la PEM observada y la predicha en la población estudiada (valor de referencia para la $\mathrm{PEM}=134,42 \pm 16,13 \mathrm{cmH}_{2} \mathrm{O}$ en mujeres y 200,25 $\pm 20,91 \mathrm{cmH}_{2} \mathrm{O}$ en hombres; tabla 2).

Tabla 1. Características de la población de estudio

\begin{tabular}{|c|c|c|}
\hline Variable & Mujeres $(\mathrm{n}=24)$ & Hombres $(\mathrm{n}=39)$ \\
\hline Edad (años) & $43,29 \pm 17,79$ & $47,56 \pm 16,04$ \\
\hline \multicolumn{3}{|l|}{ Antropometría } \\
\hline Peso $(k g)$ & $63,53 \pm 10,19$ & $82,34 \pm 11,43$ \\
\hline Altura $(\mathrm{cm})$ & $162,92 \pm 6,70$ & $173,51 \pm 6,17$ \\
\hline IMC (kg/m2) & $23,91 \pm 3,22$ & $27,34 \pm 3,54$ \\
\hline \multicolumn{3}{|l|}{ IPAQ-short } \\
\hline MET-min/sem & $2619,81 \pm 1921,41$ & $3062,96 \pm 2230,93$ \\
\hline Tiempo sentado (h/sem) & $6,55 \pm 2,48$ & $6,64 \pm 2,86$ \\
\hline \multicolumn{3}{|l|}{ Espirometría forzada } \\
\hline FEV1 (1) & $3,00 \pm 0,62$ & $3,91 \pm 0,83$ \\
\hline FEV1 $(\%)$ & $103,92 \pm 10,91$ & $104,18 \pm 14,61$ \\
\hline FVC (1) & $3,65 \pm 0,65$ & $4,92 \pm 0,94$ \\
\hline $\mathrm{FVC}(\%)$ & $98,04 \pm 8,31$ & $100,13 \pm 13,32$ \\
\hline $\mathrm{FEV}_{1} / \mathrm{FVC}$ & $82,3 \pm 7,32$ & $79,08 \pm 5,59$ \\
\hline $\mathrm{FEV}_{1} / \mathrm{FVC}(\%)$ & $105,38 \pm 7,20$ & $103,08 \pm 6,26$ \\
\hline $\operatorname{PEF}(1 / \mathrm{s})$ & $6,75 \pm 1,24$ & $8,96 \pm 1,68$ \\
\hline $\operatorname{PEF}(\%)$ & $103,17 \pm 13,66$ & $95,38 \pm 16,03$ \\
\hline $\mathrm{FEF}_{25-75 \%}(1 / \mathrm{s})$ & $3,18 \pm 1,25$ & $3,71 \pm 1,34$ \\
\hline $\mathrm{FEF}_{25-75 \%}(\%)$ & $110,42 \pm 29,81$ & $104,79 \pm 29,46$ \\
\hline \multicolumn{3}{|c|}{ Presiones respiratorias máximas } \\
\hline $\mathrm{PIM}\left(\mathrm{cmH}_{2} \mathrm{O}\right)$ & $79,42 \pm 23,87$ & $105,92 \pm 21,73$ \\
\hline PIM $(\%)$ & $80,29 \pm 23,37$ & $81,26 \pm 17,43$ \\
\hline $\mathrm{PEM}\left(\mathrm{cmH}_{2} \mathrm{O}\right)$ & $106,29 \pm 32,48$ & $160,72 \pm 40,35$ \\
\hline PEM $(\%)$ & $79,21 \pm 21,19$ & $83,08 \pm 20,98$ \\
\hline
\end{tabular}

Valores expresados como media y DE.

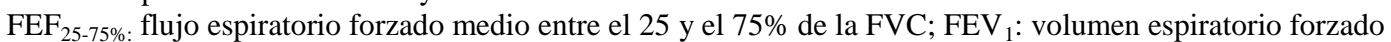
en el primer segundo; FVC: capacidad vital forzada; IMC: índice de masa corporal; IPAQ: International Physical Activity Questionnaire; 1: litros; 1/s: litros por segundo; n: número de sujetos; PEF: flujo espiratorio máximo; PEM: presión espiratoria máxima; PIM: presión inspiratoria máxima. 
Tabla 2. Comparación de las presiones respiratorias máximas en población adulta sana con los valores de referencia

\begin{tabular}{clll}
\hline & Dif. entre valor observado y VR & $\mathrm{p}$ & IC 95\% \\
\hline Mujeres $(n=24)$ & & & \\
PIM $\left(\mathrm{cmH}_{2} \mathrm{O}\right)$ & $-19,08 \pm 23,57$ & 0,001 & $-9,13(-29,04)$ \\
PEM $\left(\mathrm{cmH}_{2} \mathrm{O}\right)$ & $-28,13 \pm 29,93$ & $<0,001$ & $-15,49(-40,76)$ \\
Hombres $(n=39)$ & $-25,18 \pm 24,31$ & & \\
PIM $\left(\mathrm{cmH}_{2} \mathrm{O}\right)$ & $-39,53 \pm 44,38$ & $<0,001$ & $-17,30(-33,06)$ \\
PEM $\left(\mathrm{cmH}_{2} \mathrm{O}\right)$ & & $<0,001$ & $-25,14(-53,91)$ \\
& & & \\
\hline
\end{tabular}

Los valores son presentados como media y DE.

PEM: presión espiratoria máxima; PIM: presión inspiratoria máxima; VR: valor de referencia.

Tabla 3. Correlación entre el valor observado de PIM y PEM con diferentes variables antropométricas, espirométricas y de actividad física

\begin{tabular}{llllll}
\hline & \multicolumn{2}{c}{ PIM } & & \multicolumn{2}{c}{ PEM } \\
\cline { 2 - 3 } \cline { 5 - 6 } \cline { 5 - 5 } & Mujeres $(\mathrm{n}=27)$ & Hombres $(\mathrm{n}=39)$ & & Mujeres $(\mathrm{n}=27)$ & Hombres $(\mathrm{n}=39)$ \\
\hline Edad & $-0,026$ & $-0,320^{*}$ & & 0,176 & $-0,058$ \\
Peso & 0,170 & $-0,055$ & 0,304 & 0,185 \\
Talla & 0,205 & 0,032 & & 0,247 & 0,168 \\
IPAQ & 0,103 & $-0,375^{*}$ & 0,172 & 0,269 & $-0,288$ \\
FVC & 0,265 & 0,271 & 0,001 & 0,100 \\
FEV & 0,061 & $0,406^{*}$ & 0,265 & 0,158 \\
PEF & 0,339 & & & 0,250 \\
\hline
\end{tabular}

$\mathrm{FEV}_{1}$ : volumen espiratorio forzado en el primer segundo; FVC: capacidad vital forzada; IPAQ: International Physical Activity Questionnaire; PEF: flujo espiratorio máximo; PEM: presión espiratoria máxima; PIM: presión inspiratoria máxima.

$* \mathrm{p}<0,05$.

Por último, en la regresión cuadrática realizada para explorar el comportamiento de la diferencia entre el valor observado y el valor predicho de las PRM en relación con la edad, se observa, tanto para la PIM como para la PEM, en ambos sexos, que las ecuaciones de Morales et al. ${ }^{5}$, predicen mejor los valores observados entre los 40 y los 60 años. Sin embargo, estas ecuaciones parecen sobreestimar las PRM en sujetos por debajo de los 40 años y por encima de los 60 años (fig. 1). 

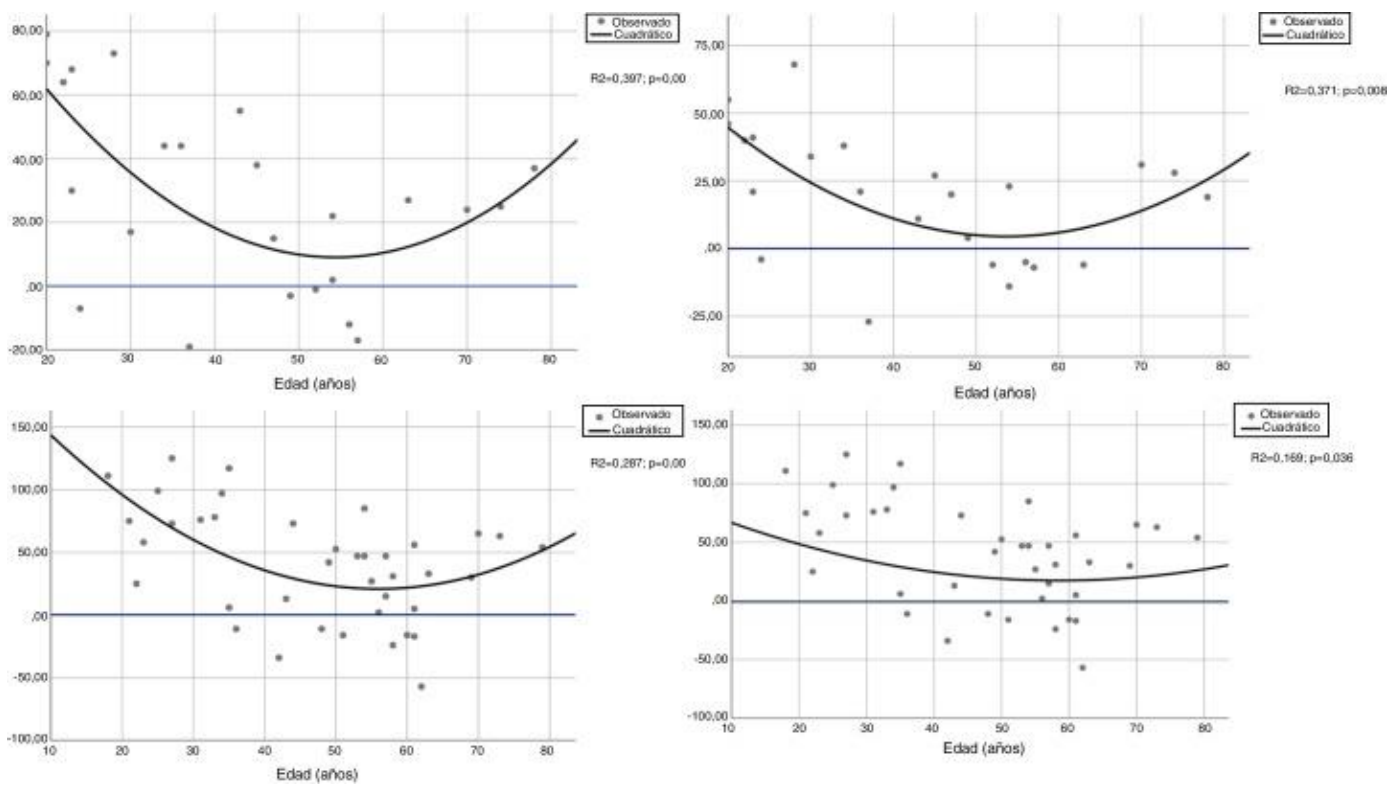

Figura 1. En la figura se presenta la regresión curvilínea que expresa la diferencia entre el valor de referencia y el valor observado para la PIM y para la PEM, en ambos sexos, en relación con la edad. La línea horizontal corresponde a la diferencia 0 , es decir, donde deberían de situarse los valores en caso de que la ecuación predictiva se ajustase perfectamente a la realidad de los valores observados en la muestra.

\section{Discusión}

Nuestros resultados demuestran que existen diferencias estadísticamente significativas entre los valores observados de las PRM y los calculados mediante las ecuaciones predictivas propuestas por Morales et al. ${ }^{5}$, en una muestra española adulta sana. Estas parecen sobreestimar los valores obtenidos tanto para hombres como para mujeres, especialmente en los varones, y sobre todo para la PEM.

Las diferencias en las PRM halladas entre el valor observado en nuestra muestra y las propuestas como teóricas por Morales et al. ${ }^{5}$, no pueden ser explicadas en base a las características de la muestra, ya que en ambos estudios, las principales variables que determinan su valor, según las ecuaciones predictivas, son equiparables. La edad media global fue de 45,94 $\pm 16,71$ años en nuestra muestra y de $49 \pm 18$ en el estudio de Morales et al. ${ }^{5}$, siendo los rangos mínimo y máximo de edad también equiparables. Asimismo, las variables antropométricas fueron similares.

Una de las principales diferencias que existe entre el estudio de Morales et al. ${ }^{5}$ y el nuestro es la procedencia de la población reclutada. En ambos trabajos se trata de población española, pero procedente de diferentes zonas geográficas (Comunidad Valenciana vs. Galicia). Pueden existir diferencias de carácter sociocultural, como la actividad física y la alimentación, que a su vez influyan en el estado muscular y en las presiones máximas que son capaces de generar los individuos con su musculatura respiratoria. Si aceptáramos esta particularidad geográfica, significaría que las ecuaciones de Morales solo podrían utilizarse en la región mediterránea del país. Este es el primer motivo que sugiere la necesidad de nuevas ecuaciones de referencia que incluyan una muestra representativa de toda la geografía española. Por otro lado, es importante recordar que la ERS recomienda que los valores de referencia relacionados con la función pulmonar sean actualizados cada 10 años ${ }^{12}$. 
Con respecto al protocolo de medición empleado, existe gran diversidad en la literatura, destacando la falta de acuerdo entre el protocolo propuesto por la ATS/ERS ${ }^{8}$ y el propuesto por la $\mathrm{SEPAR}^{9}$, en cuanto al tiempo de duración de la maniobra, el uso de pinzas nasales, el número de maniobras totales realizadas y su reproductibilidad. Diferentes estudios han puesto de manifiesto que cambios en el protocolo de medición pueden suponer variaciones sustanciales en los valores registrados ${ }^{10}$. No obstante, en nuestro estudio se empleó el mismo protocolo de medición que el detallado en el artículo de Morales et al. ${ }^{5}$, el cual está en consonancia con la normativa vigente de la SEPAR para la determinación de las PRM $^{9}$ facilitando la comparación de los valores obtenidos con los calculados mediante la ecuación.

Otro aspecto relevante son los avances tecnológicos en salud producidos en el transcurso de los últimos 20 años que han dado lugar a nuevos equipos de medición que podrían aportar mayor precisión en la determinación de las PRM. De igual manera, en las últimas décadas las herramientas computacionales han permitido un gran desarrollo del análisis estadístico, que facilita actualmente generar modelos multidimensionales, aplicables a la formulación de nuevas ecuaciones de referencia. En consecuencia, esto permite pensar que a partir de un estudio poblacional adecuado, utilizando dispositivos de medición modernos y aplicando un análisis estadístico actual, podríamos obtener unas ecuaciones más fiables y reproducibles.

Por último, se analizó la existencia de relación lineal entre la PIM y la PEM para ambos sexos con las siguientes variables: edad, medidas antropométricas, actividad física y los principales parámetros espirométricos. Se observó que existe una correlación ligera entre el PEF y la actividad física con la PIM para los hombres, y una correlación moderada para el mismo sexo entre la PIM y la edad. En el trabajo de Morales et al. ${ }^{5}$, no se halló correlación entre las PRM y la actividad física. Si bien otros autores objetivaron esta variable mediante cuestionarios validados para este mismo tipo de estudios ${ }^{21}$, consideramos que se podría afinar más en su evaluación, por ejemplo, con dispositivos de acelerometría, que han demostrado ser fiables y reproducibles para poder definir con mayor precisión qué papel juega el nivel de actividad física sobre el valor de las PRM ${ }^{22}$. Por otra parte, es importante destacar que en nuestro estudio la edad tan solo demostró una ligera correlación con la PIM en el caso de los hombres y, sin embargo, es el único factor de la ecuación predictiva de Morales et al. ${ }^{5}$, que interactúa junto con el peso en la predicción de la PIM en varones y de la PEM en mujeres. Otras ecuaciones a nivel mundial han tomado únicamente la edad como factor que explica las variables PIM y PEM; tal es el caso de las propuestas por Black y Hyatt $^{11}$, del año 1969, empleadas habitualmente en España cuando todavía no existían las de Morales et al. ${ }^{5}$. Otros ejemplos los encontramos en población brasileña ${ }^{23,24} \mathrm{y}$ en población de India $^{25}$. Las ecuaciones de Simões et al. ${ }^{21}$, para población brasileña también contemplan únicamente la edad para la predicción de ambas presiones en los hombres y la edad y el peso en el caso de las mujeres. Sin embargo, otros autores han propuesto ecuaciones con diferentes variables, como es el caso de las ecuaciones de Wilson et al. ${ }^{26}$, realizadas en Reino Unido en el año 1984, en las cuales se introduce la variable estatura para la predicción de la PIM y la PEM en las mujeres. En el caso de las ecuaciones predictivas para la PIM de Harik-Khan et al. ${ }^{27}$, para sujetos de Baltimore, estas están en función de la edad y el peso en el caso de los varones, y para las mujeres, además de las anteriores, se incluye también la talla.

En relación a la influencia que podría tener la edad para explicar las variables PIM y PEM, en ambos sexos, si bien el reducido tamaño muestral de nuestro estudio es un factor limitante, los hallazgos presentados señalan la necesidad de explorar nuevas ecuaciones de referencia distintas para diferentes franjas etarias, ya que hemos observado que las ecuaciones predictivas propuestas por Morales et al..$^{5}$, predicen mejor los valores en sujetos de 40 a 60 años, y sin embargo, estas mismas ecuaciones parecen no ajustarse a la realidad de los valores obtenidos en sujetos por debajo de los 40 y por encima de los 60 años (fig. 1). 
La magnitud de las diferencias halladas en el presente estudio entre los valores observados y los valores predichos a través de las ecuaciones de referencia de las PRM tiene gran relevancia clínica si analizamos los datos atendiendo al intervalo de confianza al 95\%. Así, observamos que en el caso de los hombres, existe una diferencia para las PRM con rangos entre -17 y $-53 \mathrm{cmH}_{2} \mathrm{O}$ y para las mujeres entre -9 y $-41 \mathrm{cmH}_{2} \mathrm{O}$ (tabla 2). Esta gran variabilidad podría suponer que una persona con una fuerza de la musculatura respiratoria dentro de los rangos de normalidad se diagnosticase erróneamente como debilidad muscular. Asimismo, estas diferencias son clínicamente relevantes desde el momento que condicionan la pauta para dosificar la carga de entrenamiento de la musculatura respiratoria en pacientes con debilidad.

\section{Conclusiones}

El presente estudio muestra que existen diferencias estadísticamente significativas entre las presiones respiratorias máximas observadas en una muestra de sujetos españoles adultos sanos y las obtenidas a través de las ecuaciones predictivas propuestas por Morales en el año 1997, sobreestimando estas el valor real hallado en la población.

Este trabajo pone de manifiesto la necesidad de realizar un estudio multicéntrico, con población representativa de todo el territorio nacional, que permita establecer nuevas ecuaciones de referencia en concordancia con el valor real de las PRM a fin de poder implementar su uso tanto en el ámbito clínico como en investigación.

\section{Conflicto de intereses}

Los autores declaran no tener ningún conflicto de intereses.

\section{Financiación}

Los autores declaran que el estudio se llevó a cabo sin financiación externa.

\section{Bibliografía}

1. Mendis S. Global status report on noncommunicable diseases 2014 [Internet]. Geneva: World Health Organization; 2014 [consultado 8 Mayo 2018]. Disponible en: http://apps.who.int/iris/bitstream/handle/10665/148114/97 89241564854 eng.pdf?sequence=1.

2. Global Initiative for Chronic Obstructive Lung Disease. Global strategy for the diagnosis, management, and prevention of chronic obstructive pulmonary disease [Internet]. [Vancouver, WA]: GOLD; 2018 [consultado 24 Jul 2018]. Disponible en: https://goldcopd.org/wp-content/uploads/2017/11/ GOLD-2018v6.0-FINAL-revised-20-Nov WMS.pdf.

3. Maltais F, Decramer M, Casaburi R, Barreiro E, Burelle Y, Debigaré R, et al. An official American Thoracic Society/European Respiratory Society statement: update on limb muscle dysfunction in chronic obstructive pulmonary disease. Am J Respir Crit Care Med. 2014;189:e15-62.

4. Barreiro E, Bustamante V, Cejudo P, Gáldiz JB, Gea J, de Lucas P, et al. Normativa SEPAR sobre disfunción muscular de los pacientes con Enfermedad Pulmonar Obstructiva Crónica. Arch Bronconeumol. 2015;51:384-95.

5. Morales P, Sanchis J, Cordero PJ, Díez JL. Presiones respiratorias estáticas máximas en adultos: valores de referencia de una población caucasiana mediterránea. Arch Bronconeumol. 1997;33:213-9.

6. Domínguez Flores ME, Luna Padrón E, Peñalosa Ochoa L, Galicia Amor S, Toral Freyre S, Baños, Mejía BO, et al. Guía para el diagnóstico y tratamiento de las alteraciones respiratorias en las enfermedades neuromusculares. Neumol Cir Tórax. 2011;70:5-70. 
7. Gomes Neto M, Saquetto M, Silva C, Carvalho V, Ribeiro N, Conceição C. Effects of respiratory muscle training on respiratory function respiratory muscle strength, and exercise tolerance in patients poststroke: a systematic review with meta-analysis. Arch Phys Med Rehabil. 2016;97: 1994-2001.

8. ATS/ERS statement on respiratory muscle testing. Am J Respir Crit Care Med. 16642002 518-624.

9. Calaf N. Medición de las presiones respiratorias máximas En: Manual SEPAR de procedimientos.: Procedimientos de evaluación de la función pulmonar II [Internet], 4. Barcelona: Sociedad Española de Neumología y Cirugía Torácica; 2004. p. 134-44 [consultado 8 May 2018]. Disponible en: http://issuu.com/separ/docs/procedimientos4?e = 3049452/25 68662 .

10. Sclauser Pessoa IM, Franco Parreira V, Fregonezi GA, Sheel AW, Chung F, Reid WD. Reference values for maximal inspiratory pressure: a systematic review. Can Respir J. 2014;21:43-50.

11. Black LF, Hyatt RE. Maximal respiratory pressures: normal values and relationship to age and sex. Am Rev Respir Dis. 1969;99:696-702.

12. Pellegrino R, Viegi G, Brusasco V, Crapo RO, Burgos F, Casaburi R, et al. Interpretative strategies for lung function test. Eur Respir J. 2005;26:948-68.

13. Araújo PR, Resqueti V, Nascimento Junior J, Carvalho LdA, Cavalcanti AG, Silva V, et al. Reference values for sniff nasal inspiratory pressure in healthy subjects in Brazil: a multicenter study. J Bras Pneumol. 2012;38:700-7.

14. Casan P, Burgos F, Barberà JA, Giner J. Espirometría En: Manual SEPAR de procedimientos. Vol. 3: Procedimientos de evaluación de la función pulmonar I [Internet]. Barcelona: Sociedad Española de Neumología y Cirugía Torácica; 2002. p. 4-15 [consultado 8 May 2018]. Disponible en: http://issuu.com/separ/docs/procedimientos $3 ?=3049452 / 2571870$

15. García-Río F, Calle M, Burgos F, Casan P, del Campo F, Galdiz JB, et al. Espirometría. Arch Bronconeumol. 2013;49:388-401.

16. Craig C, Marshall A, Sjöström M, Bauman A, Booth M, Ainsworth B, et al. International physical activity questionnaire: 12-country reliability and validity. Med Sci Sports Exerc. 2003;35:1381-95.

17. Miller MR, Crapo R, Hankinson J, Brusasco V, Burgos F, Casaburi R, et al. General considerations for lung function testing. Eur Respir J. 2005;26:153-61.

18. Miller MR, Hankinson J, Brusasco V, Burgos F, Casaburi R, Coates A, et al. Standardisation of spirometry. Eur Respir J. 2005;26:319-38.

19. Terzi N, Corne F, Mouadil A, Lofaso F, Normand H. Mouth and nasal inspiratory pressure: learning effect and reproducibility in healthy adults. Respiration. 2010;80:379-86.

20. Dancey C, Reidy J. Statistics without maths for psychology: using SPSS for Windows. London: Prentice Hall; 2004.

21. Simões R, Deus AP, Auad M, Dionísio J, Mazzonetto M, Borghi Silva A. Maximal respiratory pressure in healthy 20 to 89 yearold sedentary individuals of central São Paulo State. Rev Bras Fisioter. 2010;14:60-7.

22. Vähä Ypyä H, Vasankari T, Husu P, Mänttäri A, Vuorimaa T, Suni J, et al. Validation of cut-points for evaluating the intensity of physical activity with accelerometry-based Mean Amplitude Deviation (MAD). PLoS ONE. 2015;10:e0134813.

23. Costa D, Gonc, alves H, de Lima L, Ike D, Cancelliero K, Montebelo MIdL. New reference values for maximal respiratory pressures in the Brazilian population. J Bras Pneumol. 2010;36: 306-12.

24. Neder JA, Andreoni S, Lerario MC, Nery LE. Reference values for lung function tests II. Maximal respiratory pressures and voluntary ventilation. Braz J Med Biol Res. 1999;32:719-27.

25. Gopalakrishna A, Vaishali K, Prem V, Aaron P. Normative values for maximal respiratory pressures in an Indian Mangalore population: A cross-sectional pilot study. Lung India. 2011;28:247-52.

26. Wilson SH, Cooke NT, Edwards RH, Spiro SG. Predicted normal values for maximal respiratory pressures in caucasian adults and children. Thorax. 1984;39:535-8.

27. Harik Khan RI, Wise RA, Fozard JL. Determinants of maximal inspiratory pressure The Baltimore Longitudinal Study of Aging. Am J Respir Crit Care Med. 1998;158:1459-64. 